\title{
FORMULæ FOR SUMS INVOLVING A REDUCED SET OF RESIDUES MODULO $n$
}

\author{
by E. SPENCE
}

(Received 30th September 1963)

In this note we prove the following result:

If $n$ is a positive integer $>1, m$ the square-free part of $n$, and if

$$
1=a_{1}<a_{2}<\ldots<a_{\phi(n)}=n-1
$$

are the positive integers less than $n$, relatively prime to $n$, then

$$
\sum_{j=1}^{\phi(n)} j a_{j}=\frac{\phi(n)}{24}\left\{8 n \phi(n)+6 n+2 \phi(m)(-1)^{\omega(m)}-2^{\omega(m)}\right\},
$$

where $\omega(m)$ is the number of prime factors of $m$.

To prove this result we consider first the case when $n$ itself is square-free, $n=p_{1} p_{2} \ldots p_{r}$ where the $p_{i}$ are distinct primes, $i=1,2, \ldots, r$. For every integer $k$, let $M(n, k)$ be the number of integers $a_{i}$ in the set (1) such that $\left(a_{i}+k, n\right)=1$. (In the general case when $n$ is not necessarily square-free, $M(n, k)$ is Nagell's totient function. For a discussion of this function see Alder (1).) By considering the $r$ congruences

$$
x_{i}+k \equiv 0\left(\bmod p_{i}\right)
$$

where $x_{i} \not \equiv 0\left(\bmod p_{i}\right), i=1,2, \ldots, r$, it follows easily that

$$
M(n, k)=\prod_{\substack{p|k \\ p| n}}(p-1) \prod_{\substack{p \nmid k \\ p \mid n}}(p-2)=\phi(n) \prod_{\substack{p \nmid k \\ p \mid n}} \frac{p-2}{p-1} .
$$

Now let $(k, n)=d_{k}$; then since $n$ is square-free, $p \mid n$ and $p \nmid k$ if, and only if $p \mid\left(n / d_{k}\right)$, and hence

$$
M(n, k)=\phi(n) \prod_{p \mid \frac{n}{d_{k}}} \frac{p-2}{p-1} .
$$

If $d$ is square-free, let $\psi(d)=\prod_{p \mid d}(p-2)(d>1), \psi(1)=1$. Then $\psi$ is multiplicative on the square-free integers, and

$$
M(n, k)=\phi(n) \psi\left(n / d_{k}\right) / \phi\left(n / d_{k}\right)=\phi\left(d_{k}\right) \psi\left(n / d_{k}\right) .
$$

Now let $s=\phi(n)$ and consider the set of integers

$$
a_{1}, a_{2}, \ldots, a_{s}, a_{s+1}, \ldots, a_{2 s} \text {, }
$$


where $a_{s+i}=n+a_{i}, i=1,2, \ldots, s$. From the above, if $1 \leqq k \leqq n, M(n, k)$ is the number of pairs $\left(a_{i}, a_{j}\right)$ in this set such that

with $j \leqq s$. Hence

$$
a_{i}-a_{j}=k
$$

$$
\sum_{j=1}^{s} \sum_{i=1}^{s}\left(a_{i+j}-a_{j}\right)^{2}=\sum_{k=1}^{n} k^{2} M(n, k)=\sum_{k=1}^{n} k^{2} \phi\left(d_{k}\right) \psi\left(n / d_{k}\right) .
$$

Now suppose that $d \mid n$; then $d=d_{k}$ if, and only if $k=d$ (an integer relatively prime to $n / d$ ). Thus, if $G(n)$ denotes the sum of the squares of the positive integers less than $n$, relatively prime to $n$, then

$$
\begin{aligned}
\sum_{k=1}^{n} k^{2} M(n, k) & =\sum_{d \mid n} d^{2} G(n / d) \phi(d) \psi(n / d) \\
& =n^{2} \phi(n) \sum_{d \mid n} \frac{G(d)}{d^{2}} \frac{\psi(d)}{\phi(d)}
\end{aligned}
$$

Now it is easy to prove that if $m$ is square-free, then

$$
G(m)=\frac{1}{6} m \phi(m)\left(2 m+(-1)^{\omega(m)}\right), \quad m>1 .
$$

(See for example, Nagell (2) Ex. 35, Ch. I.) Hence

$$
\begin{aligned}
\sum_{k=1}^{n} k^{2} M(n, k) & =\frac{1}{6} n^{2} \phi(n) \sum_{\substack{d \prod_{d} n \\
d>1}} \frac{\psi(d)}{d}\left(2 d+(-1)^{\omega(d)}\right)+n^{2} \phi(n) \\
& =\frac{1}{3} n^{2} \phi(n)(\phi(n)-1)+\frac{1}{6} n^{2} \phi(n)\left(\frac{2^{\omega(n)}}{n}-1\right)+n^{2} \phi(n) \\
& =\frac{1}{6} n^{2} \phi(n)(2 \phi(n)+3)+\frac{1}{6} n \phi(n) 2^{\omega(n)}
\end{aligned}
$$

The second of these equalities follows from the fact that in the first sum each of the terms is multiplicative (on the square-free integers).

Using the following results, which are easily verified,

$$
\begin{aligned}
\sum_{j=1}^{s} \sum_{i=1}^{s}\left(a_{i+j}-a_{j}\right)^{2} & =\sum_{j=1}^{s} \sum_{i=1}^{s-j}\left(a_{i+j}-a_{j}\right)^{2}+\sum_{j=1}^{s} \sum_{i=j+1}^{s}\left(a_{i+j}-a_{j}\right)^{2} \\
& =\sum_{j=1}^{s} \sum_{i=j+1}^{s}\left(a_{i}-a_{j}\right)^{2}+\sum_{j=1}^{s} \sum_{i=1}^{j}\left(a_{s+i}-a_{j}\right)^{2} \\
& =\sum_{j=1}^{s} \sum_{i=1}^{s}\left(a_{i}-a_{j}\right)^{2}+\frac{n^{2}}{2} s(s+1)+2 n \sum_{j=1}^{s} \sum_{i=1}^{j}\left(a_{i}-a_{j}\right), \\
& \sum_{j=1}^{s} \sum_{i=1}^{s}\left(a_{i}-a_{j}\right)^{2}=\frac{n s^{2}}{6}\left(n+2(-1)^{\omega(n)}\right), \\
& \sum_{j=1}^{s} \sum_{i=1}^{j}\left(a_{i}-a_{j}\right)=\frac{n}{2} s(s+1)-2 \sum_{j=1}^{s} j a_{j},
\end{aligned}
$$


we deduce that

Thus

$$
\sum_{k=1}^{n} k^{2} M(n, k)=\frac{1}{6} n s^{2}\left(n+2(-1)^{\omega(n)}\right)+\frac{3}{2} n^{2} s(s+1)-4 n \sum_{j=1}^{s} j a_{j} .
$$

$$
4 n \sum_{j=1}^{s} j a_{j}=\frac{3}{2} n^{2} s(s+1)+\frac{1}{6} n s^{2}\left(n+2(-1)^{\omega(n)}\right)-\frac{1}{6} n s 2^{\omega(n)}-\frac{1}{6} n^{2} s(2 s+3)
$$

which, after simplification, yields

$$
\sum_{j=1}^{\phi(n)} j a_{j}=\frac{\phi(n)}{24}\left\{8 n \phi(n)+6 n+2 \phi(n)(-1)^{\omega(n)}-2^{\omega(n)}\right\}
$$

Now let $n$ be an arbitrary integer $>1$ and let $m$ be its square-free part. If $1=b_{1}<b_{2}<\ldots<b_{\phi(m)}=m-1$ are the positive integers less than $m$ relatively prime to $m$, then the integers $b_{J}+l m, j=1,2, \ldots, \phi(m), l=0,1$, $\ldots, \frac{n}{m}-1$, are all the positive integers less than $n$ relatively prime to $n$. Thus if these integers are denoted by $a_{1}<a_{2}<\ldots<a_{\phi(n)}$, then

$$
\sum_{j=1}^{\phi(n)} j a_{j}=\sum_{j=1}^{\phi(m)} \sum_{l=0}^{\frac{n}{m}-1}(j+l \phi(m))\left(b_{j}+l m\right) .
$$

It is now an easy matter to deduce from this the result stated earlier for arbitrary integers $>1$.

Corollary 1. In the same notation,

$$
\sum_{j=1}^{\phi(n)} j^{2} a_{j}=\frac{\phi(n)}{24}(\phi(n)+1)\left\{6 n \phi(n)+2 n+2 \phi(m)(-1)^{\omega(m)}-2^{\omega(m)}\right\} .
$$

\section{Corollary 2.}

$$
\sum_{j=1}^{\phi(n)} j a_{j}^{2}=\frac{\phi(n)}{24}\left\{6 n^{2} \phi(n)+4 n^{2}+2 m(-1)^{\omega(m)}(2 \phi(n)+1)-n 2^{\omega(m)}\right\} .
$$

The proofs are straightforward and follow from the identity

$$
\sum_{j=1}^{\phi(n)} j^{\alpha} a_{j}^{\beta}=\sum_{j=1}^{\phi(n)}(\phi(n)+1-j)^{\alpha}\left(n-a_{j}\right)^{\beta}
$$

$\alpha=2, \beta=1$ gives corollary 1 , and $\alpha=1, \beta=2$, together with the fact that

$$
G(n)=\frac{1}{6} \phi(n)\left(2 n^{2}+m(-1)^{\omega(m)}\right)
$$

for arbitrary integers $n>1$, yields corollary 2 .

\section{REFERENCES}

(1) H. L. Alder, A generalisation of the Euler $\phi$-function, Amer. Math. Monthly, 65 (1958), 690-692.

(2) T. NAGELL, Introduction to Number Theory (Uppsala, 1950).

THE UNIVERSITY

GLASGOW 